\title{
Poils movillés : une expérience de recherche et de partage de savoirs... à hérisser les cheveux
}

Projet expérimental mis au point pour l'exposition interactive sur les forces et les formes dans la matière, « Ruptures », présentée au Palais de la découverte jusqu'au 10 novembre 2013 (voir annonce, p. 9).

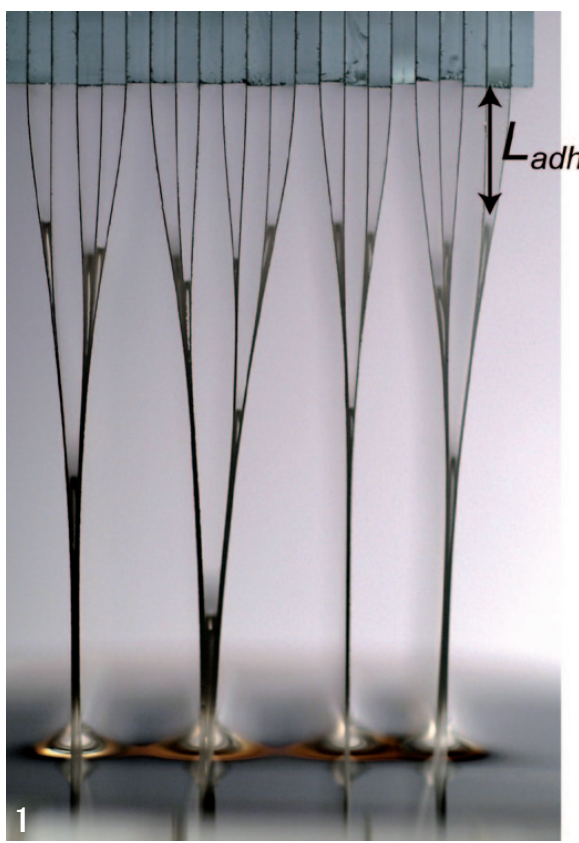

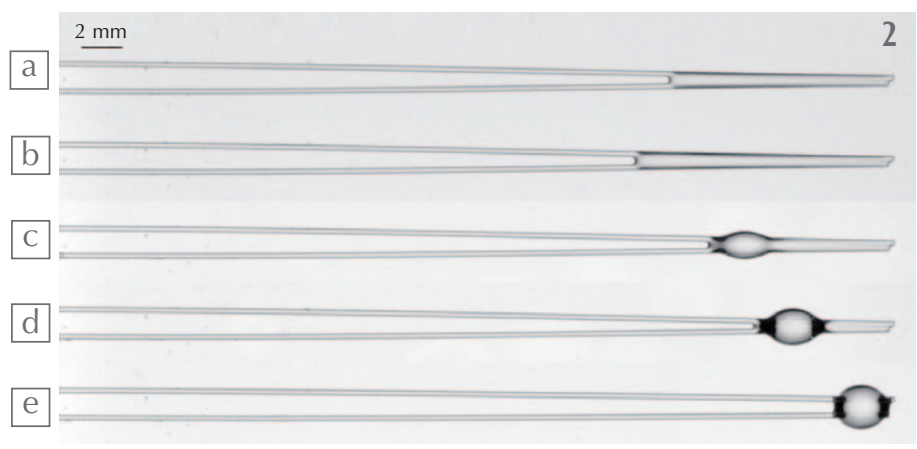

1. Retrait d'une brosse modèle d'un bain de liquide mouillant (bain d'huile, en bas de l'image). La brosse est constituée de lamelles de film polymère de $50 \mu \mathrm{m}$ d'épaisseur, séparées de $1 \mathrm{~mm}$ par des lames de microscope (qui apparaissent bleutées en haut de l'image). Les lamelles s'assemblent successivement en des mèches de plus en plus grosses, selon un processus de coalescence capillaire.

2. Détermination du volume de liquide optimal permettant le collage de deux fibres (le volume de liquide croît du haut vers le bas). Dans un premier temps, l'augmentation du volume de liquide accroît l'extension de la zone collée (a, b). Cependant, au-delà d'un volume optimal, le long pont capillaire se déstabilise en une goutte de petit diamètre et les fibres se décollent (c, d, e).

\section{Références}

1• J. Bico, B. Roman, L. Moulin et A. Boudaoud, “Elastocapillary coalescence in wet hair", Nature, 432 (2004) 690

2• C. Duprat, S. Protière, A.Y. Beebe et H. A. Stone, "Wetting of flexible fiber arrays", Nature, 482 (2012) 510.
Trempons un vieux pinceau ébouriffé dans un verre d'eau. Une fois immergé, son état ne change guère. Retirons-le de son bain, il reprend une belle forme effilée. De la même manière, une fois mouillée, une chevelure en brosse s'associe en mèches. Cette expérience banale est importante pour des applications qui mettent en jeu des fibres (cosmétiques, matériaux composites). Elle est également cruciale lors de la mise au point de microsystèmes électromécaniques (MEMS), car une des étapes de leur élaboration implique souvent le retrait d'un bain de solvant. Les micro-ailettes d'un accéléromètre destiné à déclencher un airbag peuvent ainsi se retrouver dramatiquement collées entre elles.

Combien de cheveux se retrouvent associés dans une mèche humide ? Et quelle quantité d'eau optimise cette adhésion ? La réponse à ces questions résulte d'un équilibre entre les forces capillaires dues au liquide emprisonné et la rigidité du cheveu qui s'oppose à sa déformation. Des expériences macroscopiques sur des brosses modèles ont permis à deux groupes, à Paris (PMMH-ESPCI, LPS-ENS) et à Princeton (Dep. of Mech. and Aero. Eng.), d'élucider respectivement ces deux points. L'association en mèches résulte d'une succession de collages de paires de mèches de taille de plus en plus importante (fig. 1). L'étude d'une étape élémentaire de cette coalescence (brosse à deux poils) montre que la distance de collage $L_{a d h}$ de deux mèches par rapport à leur racine suit une loi géométrique simple :

$L_{a d h} \sim\left(d L_{e c}\right)^{1 / 2}$, où $d$ est la distance qui sépare les deux mèches et $L_{e c}$ est une longueur élastocapillaire qui compare capillarité et rigidité de la structure [1].

Une fibre possède cependant une autre échelle caractéristique : son rayon. Camille Duprat et ses collaborateurs ont ainsi montré que cet ingrédient supplémentaire dicte le volume optimal permettant le collage de deux fibres (fig. 2) [2].

Les différents auteurs de ces travaux, C. Duprat, B. Roman, J. Bico, A. Boudaoud (qui sont loin d'être des poules mouillées !), sont associés à l'exposition "Ruptures : les matériaux roulent des mécaniques" au Palais de la découverte, qui propose une cinquantaine d'expériences originales provenant d'une douzaine de laboratoires, et initialement mises en place par Centre Sciences (à Orléans, sous le titre "Toucher, casser, couler»).

José Bico (jbico@pmmh.espci.fr) PMMH, ESPCI, 10 rue Vauquelin, 75231 Paris Cedex 05 